\title{
Analysis of the Grip Strength Data Using Anti-Diagonal Symmetry Models
}

\author{
Kiyotaka Iki \\ Department of Information Sciences, Tokyo University of Science, Noda, Japan \\ Email: iki@is.noda.tus.ac.jp
}

Received 6 June 2016; accepted 2 August 2016; published 5 August 2016

Copyright (C) 2016 by author and Scientific Research Publishing Inc.

This work is licensed under the Creative Commons Attribution International License (CC BY). http://creativecommons.org/licenses/by/4.0/

c) (i) Open Access

\begin{abstract}
For the analysis of square contingency tables with the same row and column ordinal classifications, this article proposes new models which indicate the structures of symmetry with respect to the anti-diagonal of the table. Also, this article gives a simple decomposition in $3 \times 3$ contingency table using the proposed models. The proposed models are applied to grip strength data.
\end{abstract}

\section{Keywords}

Anti-Diagonal, Decomposition, Grip Strength Data, Square Contingency Table, Symmetry

\section{Introduction}

Consider the data in Table 1 . Table 1 is the data of grip strength of 805 male examinees aged 15 - 18 at high schools in Japan, which visited Tokyo University of Science, Open Campus, August, examined in 2011-2015. In Table 1 the row variable is the right hand muscle strength level and the column variable is the left hand muscle strength level. The category in Table 1 means muscle strength level compared with other people of one's age and sex. Generally, for such data with similar classifications, many observations tend to fall (or near) the main diagonal cells. For the data in Table 1, 73\% of observations concentrate in the main diagonal. Thus, the independence between classifications is unlikely to hold. Therefore, we are interested in whether or not there is a structure of symmetry with respect to the main diagonal in the table.

For the analysis of an $r \times r$ square contingency table with the same ordinal row and column classifications, let $p_{i j}$ denote the probability that an observation will fall in the ith row and $j$ th column of the table ( $i=1, \cdots, r ; j=1, \cdots, r)$. Bowker [1] proposed the symmetry model, defined by

$$
p_{i j}=\psi_{i j} \quad(i=1, \cdots, r ; j=1, \cdots, r),
$$

where $\psi_{i j}=\psi_{j i}$ (see also Martin and Pardo [2]; Kolassa and Bhagavatula [3]; Tahata and Tomizawa [4]). This 
Table 1. Grip strength test of 805 male examinees aged 15 - 18 at high schools in Japan, examined in 2011-2015. (The parenthesized values are MLEs of expected frequencies under the AMH model).

\begin{tabular}{|c|c|c|c|c|}
\hline \multirow{2}{*}{$\begin{array}{l}\text { Right hand grip } \\
\text { strength level }\end{array}$} & \multicolumn{3}{|c|}{ Left hand grip strength level } & \multirow{2}{*}{ Total } \\
\hline & Excellent (1) & Good (2) & Poor (3) & \\
\hline \multirow[t]{2}{*}{ Excellent (1) } & 74 & 89 & 3 & 166 \\
\hline & $(77.00)$ & (85.90) & $(3.00)$ & (165.90) \\
\hline \multirow[t]{2}{*}{ Good (2) } & 10 & 444 & 93 & 547 \\
\hline & $(10.81)$ & $(444.00)$ & $(96.48)$ & (551.29) \\
\hline \multirow[t]{2}{*}{ Poor (3) } & 0 & 23 & 69 & 92 \\
\hline & $(0.00)$ & (21.39) & $(66.41)$ & (87.81) \\
\hline \multirow[t]{2}{*}{ Total } & 84 & 556 & 165 & 805 \\
\hline & $(87.81)$ & (551.29) & $(165.90)$ & $(805.00)$ \\
\hline
\end{tabular}

model states that the probability that an observation will fall in the $(i, j)$ th cell of the table is equal to the probability that it falls in the $(j, i)$ th cell. Namely, this model describes a structure of symmetry with respect to the main diagonal of the table. Stuart [5] proposed the marginal homogeneity model, defined by

$$
p_{i .}=p_{\cdot i} \quad(i=1, \cdots, r) \text {, }
$$

where $p_{i}=\sum_{t=1}^{r} p_{i t}$ and $p_{\cdot i}=\sum_{s=1}^{r} p_{s i}$. This model states that the row marginal distribution is identical to the column marginal distribution. Read [6] considered the global symmetry model, defined by

$$
\sum_{i<j} \sum_{i j}=\sum_{i>j} \sum_{i j} \text {. }
$$

This model states that the probability that an observation will fall in one of the upper-right triangle cells above the main diagonal of the table is equal to the probability that it falls in one of the lower-left triangle cells below the main diagonal.

For the data in Table 1, we see that many observations fall in the upper-right triangle cells above the main diagonal. Thus, the models for symmetry between classifications are unlikely to hold. Then, the symmetry with respect to the anti-diagonal may hold for the data in Table 1. Note that the probabilities for the anti-diagonal cells are $p_{1 r}, p_{2, r-1}, \cdots, p_{r 1}$ for the $r \times r$ table. When the number of the categories is 3 , i.e., $r=3$, (such as the data in Table 1), the anti-diagonal cells are $p_{13}, p_{22}$ and $p_{31}$. Thus, we are interested in proposing new models for symmetry with respect to the anti-diagonal, which would hold for the data in Table 1.

The present paper proposes three models and gives a simple decomposition using the proposed models in $3 \times 3$ contingency table. Also it illustrates new models with the grip strength data in Table 1 .

\section{New Models and a Simple Decomposition}

Firstly, we propose a model defined by

$$
p_{i j}=\psi_{i j} \quad(i=1, \cdots, r ; j=1, \cdots, r),
$$

where $\psi_{i j}=\psi_{\text {s }^{* *} i}$. The symbol “*” denotes $i^{*}=r+1-i$. This model states that the probability that an observation will fall in the $(i, j)$ th cell of the table is equal to the probability that it falls in the $\left(j^{*}, i^{*}\right)$ th cell. Namely, this model indicates the structure of symmetry with respect to the anti-diagonal of the table. We shall refer to this model as the anti-diagonal symmetry (AS) model. Note that the AS model is a special case of the reverse conditional symmetry model, proposed by Tomizawa [7].

Secondly, we propose a model defined by

$$
\sum_{i+j<r+1} p_{i j}=\sum_{i+j>r+1} p_{i j} .
$$

Let $X$ and $Y$ denote the row and column variables, respectively. Then, this model is also expressed as

$$
\operatorname{Pr}(X+Y<r+1)=\operatorname{Pr}(X+Y>r+1) .
$$


We shall refer to this model as the anti-diagonal global symmetry (AGS) model.

Finally, we propose a model defined by

$$
p_{i \cdot}=p_{.^{*}} \quad(i=1, \cdots, r) .
$$

This model states that the row marginal distribution is identical to the column marginal distribution in reverse order. We shall refer to this model as the anti-diagonal marginal homogeneity (AMH) model.

We obtain the following theorem.

Theorem 1. When $r=3$, the AS model holds if and only if both the AGS and AMH models hold.

Proof. If the AS model holds, then the AGS and AMH models hold. Assuming that both the AGS and AMH models hold, then we shall show that the AS model holds. If the AMH and AGS models hold, then we have $p_{1 .}=p_{.3}$ (i.e., $\left.p_{11}+p_{12}=p_{23}+p_{33}\right), p_{2 .}=p_{.2}$ (i.e., $\left.p_{21}+p_{23}=p_{12}+p_{32}\right)$ and

$p_{11}+p_{12}+p_{21}=p_{23}+p_{32}+p_{33}$. Thus, we see $p_{11}=p_{33}, p_{12}=p_{23}$ and $p_{21}=p_{32}$. Namely, the AS model holds. The proof is completed.

Note that this theorem does not hold when $r \geq 4$. Let $n_{i j}$ denote the observed frequency in the $(i, j)$ th cell of the table $(i=1, \cdots, r ; j=1, \cdots, r)$ with $n=\sum \sum n_{i j}$, and let $m_{i j}$ denote the corresponding expected frequency. Assume that $\left\{n_{i j}\right\}$ have a multinomial distribution. The maximum likelihood estimates (MLEs) of $\left\{m_{i j}\right\}$ under the AS and AGS model, are expressed as the closed-forms as follows:

1) The MLE of $m_{i j}$ under the AS model is

$$
\hat{m}_{i j}= \begin{cases}\frac{n_{i j}+n_{j^{* * *}}}{2} & \left(i \neq j^{*}\right) \\ n_{i j} & \left(i=j^{*}\right) .\end{cases}
$$

2) The MLE of $m_{i j}$ under the AGS model is

$$
\hat{m}_{i j}= \begin{cases}\frac{L+R}{2 L} n_{i j} & \left(i \neq j^{*}, i+j<r+1\right) \\ \frac{L+R}{2 R} n_{i j} & \left(i \neq j^{*}, i+j>r+1\right) \\ n_{i j} & \left(i=j^{*}\right),\end{cases}
$$

where

$$
L=\sum_{i+j<r+1} n_{i j}, \quad R=\sum_{i+j>r+1} \sum_{i j} .
$$

The MLEs of $\left\{m_{i j}\right\}$ under the AMH model could be obtained using the Newton-Raphson method in the log-likelihood equation. Let $G^{2}(M)$ denote the likelihood ratio chi-squared statistic for testing goodness-of-fit of model $M$. For the AS model, $\left\{p_{i j}\right\}$ are determined by $r(r-1) / 2$ of $\left\{\psi_{i j}, i+j<r+1\right\}, r-1$ of $\left\{\psi_{i i^{*}}\right\}$ for anti-diagonal cells (since $\sum \sum p_{i j}=1$ ), thus a total of $(r+2)(r-1) / 2$. Therefore, the number of degrees of freedom (df) for testing goodness-of-fit of the AS model is $\left(r^{2}-1\right)-(r+2)(r-1) / 2=r(r-1) / 2$. Similarly, the numbers of $\mathrm{df}$ for testing goodness-of-fit of the AGS and AMH model are 1 and $r-1$, respectively. Note that when $r \geq 4$, the number of $\mathrm{df}$ for the AS model is greater than the sum of numbers of $\mathrm{df}$ for the AGS and AMH models, and when $r=3$, it is equal to the sum of them.

We shall consider the comparison between two nested models. Suppose that model $M_{2}$ is a special case of model $M_{1}$; that is, $M_{2}$ is simpler than $M_{1}$, so when $M_{2}$ holds, necessarily $M_{1}$ also holds. For testing that model $M_{2}$ holds assuming that model $M_{1}$ holds, we can use the likelihood ratio statistic $G^{2}\left(M_{2} \mid M_{1}\right)$ which is the difference between the $G^{2}\left(M_{2}\right)$ and $G^{2}\left(M_{1}\right)$. When model $M_{1}$ holds, this statistic has an asymptotic chi-squared distribution with df being equal to the difference between the $\mathrm{df}$ for $M_{2}$ and for $M_{1}$.

\section{An Example}

Consider the data in Table 1 again. All the AS, AGS and AMH models fit these data well, yielding the likelihood 
ratio statistic $G^{2}(A S)=5.53$ with $3 \mathrm{df}, G^{2}(A G S)=0.40$ with $1 \mathrm{df}$, and $G^{2}(A M H)=0.64$ with 2 df, respectively. Since the AS model is a special case of the AGS model, we shall test the hypothesis that the AS model holds assuming that the AGS model holds. Since $G^{2}(A S \mid A G S)=G^{2}(A S)-G^{2}(A G S)=5.13$ with 2 df being the difference between the numbers of $\mathrm{df}$ for the AS and AGS models, this hypothesis is accepted at the 0.05 significance level. Thus, the AS model would be preferable to the AGS model. Similarly, since the AS model is a special case of the AMH model, we shall test the hypothesis that the AS model holds assuming that the AMH model holds. Since $G^{2}(A S \mid A M H)=G^{2}(A S)-G^{2}(A M H)=4.89$ with $1 \mathrm{df}$ being the difference between the numbers of $\mathrm{df}$ for the AS and AMH models, this hypothesis is rejected at the 0.05 significance level. Therefore, the AMH model would be preferable to the AS model.

Under the AMH model, the probability that an examinee's right hand grip strength level is "Excellent (1)", is estimated to be equal to the probability that an another examinee's left hand grip strength level is "Poor (3)". Also, the probability that an examinee's right hand grip strength level is "Poor (3)", is estimated to be equal to the probability that an examinee's left hand grip strength level is "Excellent (1)".

\section{Concluding Remarks}

The decomposition of the AS model into the AGS and AMH models, given by Theorem 1, would be useful for seeing the reason for its poor fit when the AS model fits the $3 \times 3$ data poorly, and it should be considered for ordinal categorical data because all the AS, AGS and AMH models are not invariant under arbitrary same permutations of row and column categories.

\section{Acknowledgements}

The authors would like to thank the referee for their helpful comments.

\section{References}

[1] Bowker, A.H. (1948) A Test for Symmetry in Contingency Tables. Journal of the American Statistical Association, 43, 572-574. http://dx.doi.org/10.1080/01621459.1948.10483284

[2] Martin, N. and Pardo, L. (2010) A New Measure of Leverage Cells in Multinomial Loglinear Models. Communications in Statistics - Theory and Methods, 39, 517-530. http://dx.doi.org/10.1080/03610920903139991

[3] Kolassa, J.E. and Bhagavatula, H.G. (2012) Accurate Approximations to the Distribution of a Statistic Testing Symmetry in Contingency Tables. Institute of Mathematical Statistics, 8, 181-189. http://dx.doi.org/10.1214/11-imscoll812

[4] Tahata, K. and Tomizawa, S. (2014) Symmetry and Asymmetry Models and Decompositions of Models for Contingency Tables. SUT Journal of Mathematics, 50, 131-165.

[5] Stuart, A. (1955) A Test for Homogeneity of the Marginal Distributions in a Two-Way Classification. Biometrika, 42, 412-416. http://dx.doi.org/10.1093/biomet/42.3-4.412

[6] Read, C.B. (1977) Partitioning Chi-Square in Contingency Table: A Teaching Approach. Communications in Statistics-Theory and Methods, 6, 553-562. http://dx.doi.org/10.1080/03610927708827513

[7] Tomizawa, S. (1986) Four Kinds of Symmetry Models and Their Decompositions in a Square Contingency Table with Ordered Categories. Biometrical Journal, 28, 387-393. http://dx.doi.org/10.1002/bimj.4710280402 


\section{Submit or recommend next manuscript to SCIRP and we will provide best service for you:}

Accepting pre-submission inquiries through Email, Facebook, LinkedIn, Twitter, etc.

A wide selection of journals (inclusive of 9 subjects, more than 200 journals)

Providing 24-hour high-quality service

User-friendly online submission system

Fair and swift peer-review system

Efficient typesetting and proofreading procedure

Display of the result of downloads and visits, as well as the number of cited articles

Maximum dissemination of your research work

Submit your manuscript at: http://papersubmission.scirp.org/ 